\title{
Biochemical and histological study on the effect of levetiracetam on the liver and kidney of pregnant albino rats
}

\author{
W.S. Sabbah, S.M. Hanafy, M.A.A. Arafa \\ Department of Anatomy, Faculty of Medicine (Girls), Al-Azhar University, Cairo, Egypt \\ [Received: 30 May 2019; Accepted: 1 July 2019]
}

Background: Levetiracetam is a broad-spectrum antiseizure agent and one of the most commonly prescribed drugs for epilepsy. The aim of this work was to assess the effect of levetiracetam at its therapeutic range on the liver and kidney of pregnant albino rats.

Materials and methods: Forty pregnant rats were divided equally into two groups (I-II), Rats in the group I were gavaged $1.5 \mathrm{~mL} /$ day distilled water in two divided doses throughout pregnancy. Rats in the group /I were gavaged $1.5 \mathrm{~mL} /$ day distilled water (containing $36 \mathrm{mg}$ levetiracetam) in two divided doses throughout pregnancy. At the end of the experiment, blood samples were taken and the sera were separated and used for biochemical analysis. The kidneys and livers of both groups were excised and used for light and electron microscopic examination.

Results: Treatment with levetiracetam induced undesirable histopathological changes in the liver and kidney of pregnant albino rats. These changes were in the form of distortion of the hepatic architecture, dilatation of the central and the portal veins, widening of the Bowman's spaces, thickening and disruption of the glomerular basement membrane, fusion and effacement of secondary foot processes, cytoplasmic vacuolation, and swollen mitochondria with loss of their cristae. Such changes were confirmed by alteration of certain biochemical parameters related to the liver and kidney functions.

Conclusions: Levetiracetam induced deleterious effects on the liver and kidney of pregnant albino rats. Further investigations are recommended to clarify the mechanism of levetiracetam toxicity. (Folia Morphol 2019; 78, 4: 809-817)

Key words: levetiracetam, liver, kidney, pregnant rats

\section{INTRODUCTION}

Epilepsy is a common sustained disorder affecting approximately more than one million women of childbearing age [9]. The prevalence of epilepsy has been estimated to be $0.3-0.5 \%$ in pregnant women [33]. Pregnant women with epilepsy are advised to maintain antiepileptic drugs (AED) to diminish maternal and foetal disturbance joined with seizures [22].
Pharmacological treatment options of epilepsy comprise conventional AED (valproate, phenobarbital and carbamazepine), in addition to the newer second generation agents (levetiracetam, gabapentin and topiramate) [15]. Conventional AED had good therapeutic effects and low treatment cost. However, some undesired effects associated with these drugs, such as memory deterioration and Ste-

Address for correspondence: S.M. Hanafy, PhD, Department of Anatomy and Embryology, Faculty of Medicine (Girls), Al-Azhar University, Cairo, Egypt, tel: +01006902760, e-mail: sadekamin66@yahoo.com 
vens-Johnson syndrome [37]. The effectiveness of the second-generation drugs seems to be similar to that of the conventional AED. However, the newer drugs, such as levetiracetam, seem to offer a lower risk of interactions with other medications, simpler titration, and administration regimens, and improved tolerability [15].

Levetiracetam was developed as an analogue of piracetam, a drug used to improve cognitive function [24]. Levetiracetam is recognised for adjunct therapy of focal onset, primary generalised tonic-clonic, and myoclonic seizures in children and adults [25]. The correlation between binding affinity of levetiracetam analogues and their potency toward audiogenic seizures suggests that a synaptic vesicle protein, SV2A, mediates the antiseizure effects of levetiracetam [26]. Oral absorption of levetiracetam is rapid and nearly complete, with peak plasma concentrations in $1.3 \mathrm{~h}$. Two-thirds of the drug is excreted unchanged in the urine and the remainder as 2-pyrrolidone-N-butyric acid (inactive deaminated metabolite). There is no metabolism of levetiracetam in the liver as its metabolism takes place in the blood [27].

Some authors reported a significant increase in gamma glutamyl transferase in an epileptic patient on levetiracetam monotherapy [5]. Study reported some adverse effects associated with levetiracetam therapy such as sleepiness, headaches, vomiting, inflammation of the nose and throat, and irritability [28].

Data on the safety of levetiracetam during pregnancy is still limited [3]. So, this work aimed to assess the effect of levetiracetam on the liver and kidney of pregnant albino rats.

\section{MATERIALS AND METHODS}

\section{Drug}

Levetiracetam (Tiratam) was purchased from Al-Andalous for Pharmaceuticals-Egypt Industries. It was available in the form of tablets $500 \mathrm{mg}$ each.

Adult dosing can begin with $1000 \mathrm{mg}$ twice daily, increased to a maximum dosage of $3000 \mathrm{mg}$ twice daily [23]. The chosen dose was $2000 \mathrm{mg} /$ day. The equivalent dose for adult rat weighting about $200 \mathrm{~g}$ is $36 \mathrm{mg} /$ day according to reference no. [20].

\section{Animals}

Sixty adult albino rats (20 males and 40 females) of local strain weighing about $200 \mathrm{~g}$ were obtained for this study. Care of animals and practices were provided with the Animal House, Faculty of Medicine
(Girls), Al-Azhar University, Cairo, according to the Guide of the Care and Use of Laboratory Animals [11]. Each male rat was located with 2 virgin female rats in a cage for $24 \mathrm{~h}$. In the morning, the first day of pregnancy was determined by the presence of the vaginal plug [16]. Each pregnant female was placed in an individual cage until the end of study.

\section{Experimental design}

The 40 pregnant rats were divided equally into two groups:

- Group I (control) - each pregnant rat was gavaged $1.5 \mathrm{~mL} /$ day distilled water in two divided doses throughout pregnancy;

- Group II (treated) - each pregnant rat was gavaged $1.5 \mathrm{~mL} /$ day distilled water (containing $36 \mathrm{mg}$ levetiracetam) in two divided doses throughout pregnancy.

At the end of the experiment, blood samples were taken on the first day after delivery from the retro-orbital vein of all rats after ether inhalation [36]. The sera were separated by centrifugation of blood at $3000 \mathrm{rpm}$ for $15 \mathrm{~min}$, then preserved at $-20^{\circ} \mathrm{C}$ until used for biochemical analysis. Rats were sacrificed by cervical dislocation to avoid any chemical injury [6], their abdominal cavities were exposed by a midline incision, and the kidneys and livers were excised and used for light and electron microscopic examination.

\section{Histopathological preparation \\ Preparation for light microscopic examination}

Pieces of liver and kidney tissues from the control and treated groups were washed and fixed for 3 days in Bouin's solution. Later, the tissues were dehydrated in ascending graded series of ethyl alcohol and processed for paraffin wax embedding. Liver and kidney tissues blocks were cut using microtome into serial sections at a thickness of $7 \mu \mathrm{m}$. The sections were placed on glass slides and stained with haematoxylin and eosin (H\&E), Masson's trichrome for detecting collagen fibres and periodic acid Schiff (PAS reaction) for verifying the contents of carbohydrates [2]. For histopathological examination of liver and kidney tissue a microscope (LEICA DM 750) and digital camera were used.

\section{Preparation for electron microscopic examination}

Small slices of the liver and kidney tissues were fixed quickly in $2.5 \%$ phosphate-buffered glutaraldehyde ( $\mathrm{pH}$ 7.4). The specimens were then post-fixed 


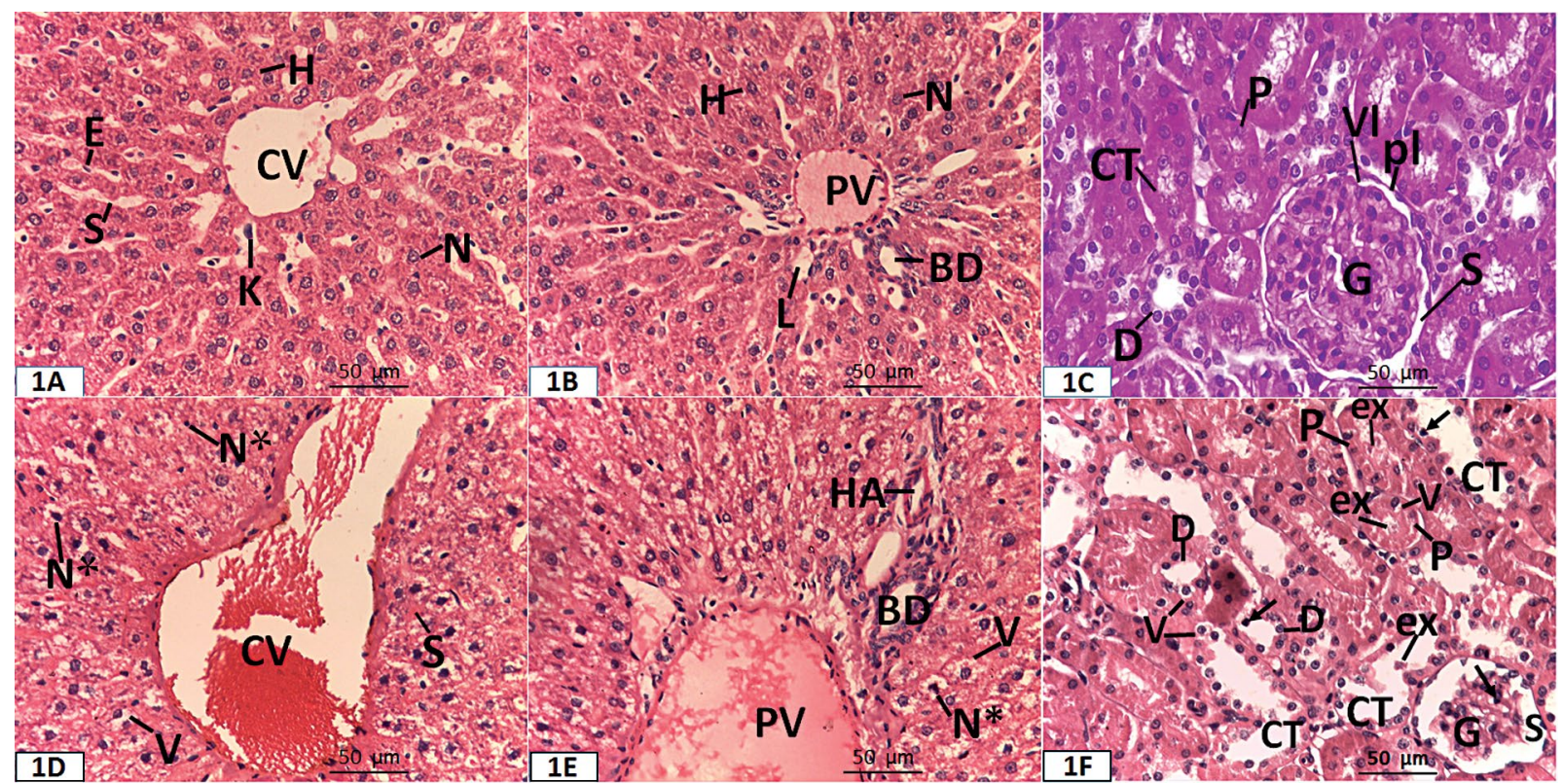

Figure 1. Liver (A, B, D, E) and renal cortex (C, F) transverse sections (H\&E $\times 400$ magnification); A. Group I shows hepatocytes $(H)$, central vein (CV), blood sinusoids (S) flat endothelial cells (E) and von Kupffer cells (K); B. Group I shows a branch of portal vein (PV), bile duct (BD) and lymph vessel (L); H - hepatocytes; N — vesicular basophilic nuclei; C. Group I shows the glomerulus (G), Bowman's space (S), the Partial (pl) and visceral (vl) layers of Bowman's capsule, proximal convoluted tubules (P), distal convoluted tubules (D) and collecting tubules (CT); D. Group II shows dilated central vein (CV), vacuolated cytoplasm (V) of hepatocytes, irregular deeply stained nuclei ( $\left.\mathrm{N}^{*}\right)$, congested blood sinusoids (S); E. Group II shows dilated portal vein (PV), vacuolated cytoplasm (V) of hepatocytes, irregular deeply stained nuclei ( $\mathrm{N}^{*}$ ), hepatic artery (HA) and bile duct (BD); F. Group II shows shrunken glomeruli (G), wide Bowman's space (BS), deeply stained glomerular cell nuclei (arrow), the lining epithelium of the PCT (P), DCT (D) and collecting tubules (CT), have vacuolated cytoplasm (V) and small deeply stained nuclei (arrow). The lumina of some renal tubules contain cellular exfoliation (ex).

with $1 \%$ osmium tetroxide in the same buffer at $41^{\circ} \mathrm{C}$, dehydrated in graded ethanol and embedded in epoxy resin [2]. Using LKB ultra-microtome, semithin sections $(0.5 \mu \mathrm{m}$ thick) were cut and stained with toluidine blue for light microscopic examination. Ultrathin sections were cut $(80 \mathrm{~nm})$, stained with uranyl acetate and lead citrate [10] and examined and photographed using electron microscope (JEOL1010 EX II, Japan) at the Regional Mycology and Biotechnology Centre, Al-Azhar University, Cairo, Egypt.

\section{Biochemical indices}

The level of some parameters of liver and kidney functions were measured using (BioSystems-BTS 310; Barcelona, Spain) which was calibrated for probes from rats.

\section{Liver function indices}

Alanine aminotransferase (ALT) and aspartate aminotransferase (AST) enzymes levels were measured in IU/L using the methods of reference no. [32].

\section{Kidney function indices}

Creatinine level in $\mathrm{mg} / \mathrm{dL}$ was determined using the method of reference no. [8]. Blood urea nitrogen measurement in $\mathrm{mg} / \mathrm{dL}$ was assessed according to reference no. [18].

\section{Statistical analysis}

Statistical analysis of the levels of ALT, AST, urea and creatinine in the studied groups were carried out using the SPSS statistical package, the data were analysed and statistical significance was determined by using unpaired Student t-test [21].

\section{RESULTS}

\section{Histopathological results \\ Liver}

Light microscopy. Examination of H\&E stained liver transverse sections of group I (control group) revealed that the liver was built up of cords of hepatocytes emitting from the central vein and spaced with blood sinusoids (Fig. 1A, B). The portal tract was built up of branches of portal vein, bile duct and 


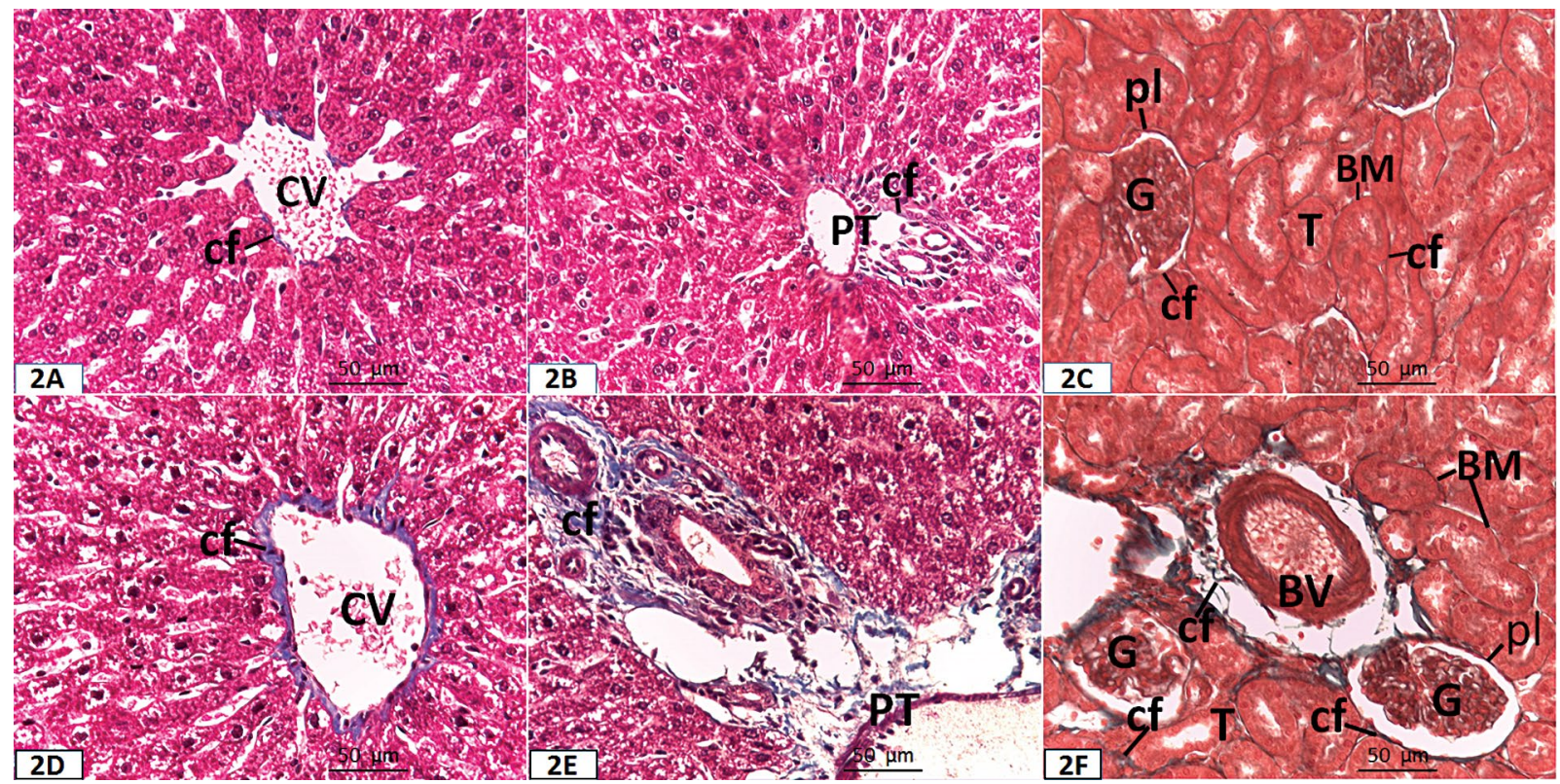

Figure 2. Liver (A, B, D, E) and renal cortex (C, F) transverse sections (Masson trichrome $\times 400$ magnification); A. Group I shows normal distribution of collagen fibres (CF) around the central vein (CV); B. Group I shows normal distribution of collagen fibres (CF) around the portal tract (PT) and between the hepatocytes; C. Group I shows the collagen fibres (cf) in the parietal layer (pl) of the Bowman's capsule, glomerular capillaries (G) and in the basement membranes (BM) of renal tubules (T); D. Group II shows a higher level of collagen fibres (CF) distribution is seen around the central vein (CV); $\mathbf{E}$. Group II shows a higher level of collagen fibre (CF) distribution is seen around the elements of the portal tract (PT); F. Group II shows increase in the collagen fibres (cf) in the parietal layer (pl) of the Bowman's capsule surrounding the glomerulus (G), in the basement membranes (BM) of renal tubules (T) and around the blood vessel (BV).

lymph vessel (Fig. 1B). Masson's trichrome staining showed minimal collagen fibres around the central vein (Fig. 2A) and the portal tract (Fig. 2B). PAS reaction revealed deposition of PAS positive material in the hepatocytes (Fig. 3A).

Inspection of H\&E stained liver transverse sections of group II (treated group) revealed that the hepatic cords did not have the normal radial arrangement, and the central vein was dilated (Fig. 1D). Most hepatocytes had cytoplasmic vacuoles, and some of their nuclei were small, irregular and deeply stained (Fig. 1D, E). The portal area showed a markedly dilated portal vein (Fig. 1E). Masson's trichrome staining showed a markedly higher level of collagen-fibre distribution around the central vein (Fig. 2D) and the elements of the portal tract as compared with sections from control group (Fig. 2E). PAS reaction showed apparent reduction of the distribution of PAS positive material in the hepatocytes (Fig. 3C).

Electron microscopy. Examination of ultrathin sections of the liver of the group I (control group) revealed that the hepatocytes had intact plasma membranes and nuclei with demarcated nuclear envelopes. The nuclei contained prominent nucleoli and both euchromatin and heterochromatin. Their cytoplasm contained rounded or oval mitochondria, free ribosome, rough endoplasmic reticulum and glycogen granules (Fig.4A).

Examination of ultrathin sections of the liver of the Group II (treated group) showed histopathological changes in the hepatocytes. Some nuclei had irregular nuclear envelopes and absent nucleoli. The cytoplasm contained vacuolated areas, few glycogen granules, swollen mitochondria with loss of the cristae and matrix, rough endoplasmic reticulum and ribosomes (Fig. 4B).

\section{Kidney}

Light microscopy. Examination of H\&E stained kidney sections of Group I (control group) showed the renal cortex was built up of Malpighian corpuscles and tubules (proximal convoluted tubules [PCT], distal convoluted tubules [DCT] and collecting tubules). The Malpighian corpuscles were formed of glomerulus surrounded by Bowman's capsule (Fig.1C). The PCT and DCT were lined by cuboidal cells. The PCT had narrow lumina with brush border on their luminal surfaces; however, the DCT exhibited more number of lining cells, and wide lumina with no brush border. The collecting tubules were lined by $10-20$ cuboidal epithelial cells (Fig.1C). 




Figure 3. Liver $(A, C)$ and renal cortex (B, D) transverse sections (PAS $\times 400$ magnification); A. Group I shows the normal distribution of PAS positive material in the hepatocytes; B. Group I shows the PAS positive reaction in the parietal layer (pl) of Bowman's capsule around the glomerulus (G), in the basement membranes (BM) of renal tubules and in the brush border (B) of the PCT (P); C. Group II shows reduction in the distribution of PAS positive material in the hepatocytes; D. Group II shows decrease in the PAS positive reaction in the parietal layer (pl) of Bowman's capsule around the glomerulus (G), in the basement membranes (BM) of renal tubules and in the brush border (B) of the PCT (P).
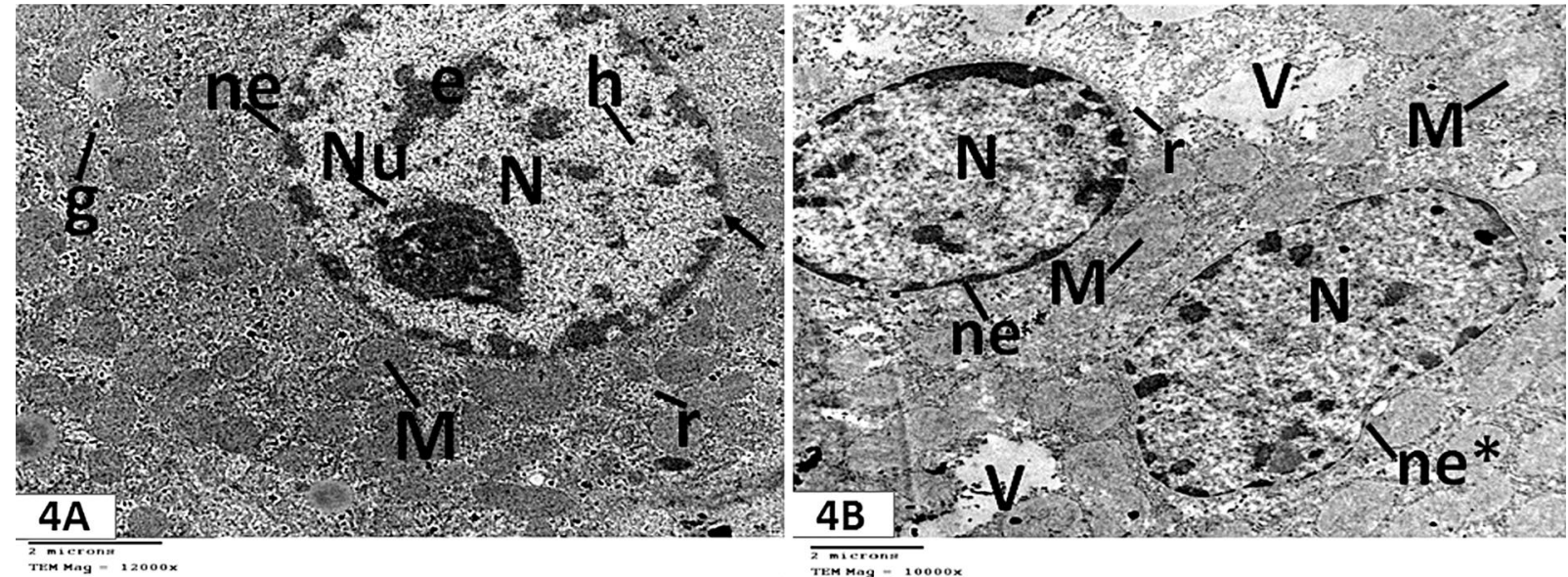

Figure 4. Transmission electron microscopy of liver sections (TEM $\times 10,000$ magnification); A. Groups I shows the cytoplasm of hepatocyte contains mitochondria $(\mathrm{M})$, ribosomes $(\mathrm{r})$, and glycogen granules $(\mathrm{g})$. Notice the nucleus $(\mathrm{N})$, nuclear envelope (ne), nuclear pores (arrow), heterochromatin (h), euchromatin (e) and prominent nucleolus (nu); B. Groups II shows the nuclei of hepatocytes (N) have irregular nuclear envelopes ( $\left(\mathrm{e}^{*}\right)$ and absent nucleoli. The cytoplasm contains vacuolated areas (V), mitochondria with a loss of cristae (M), ribosomes. 


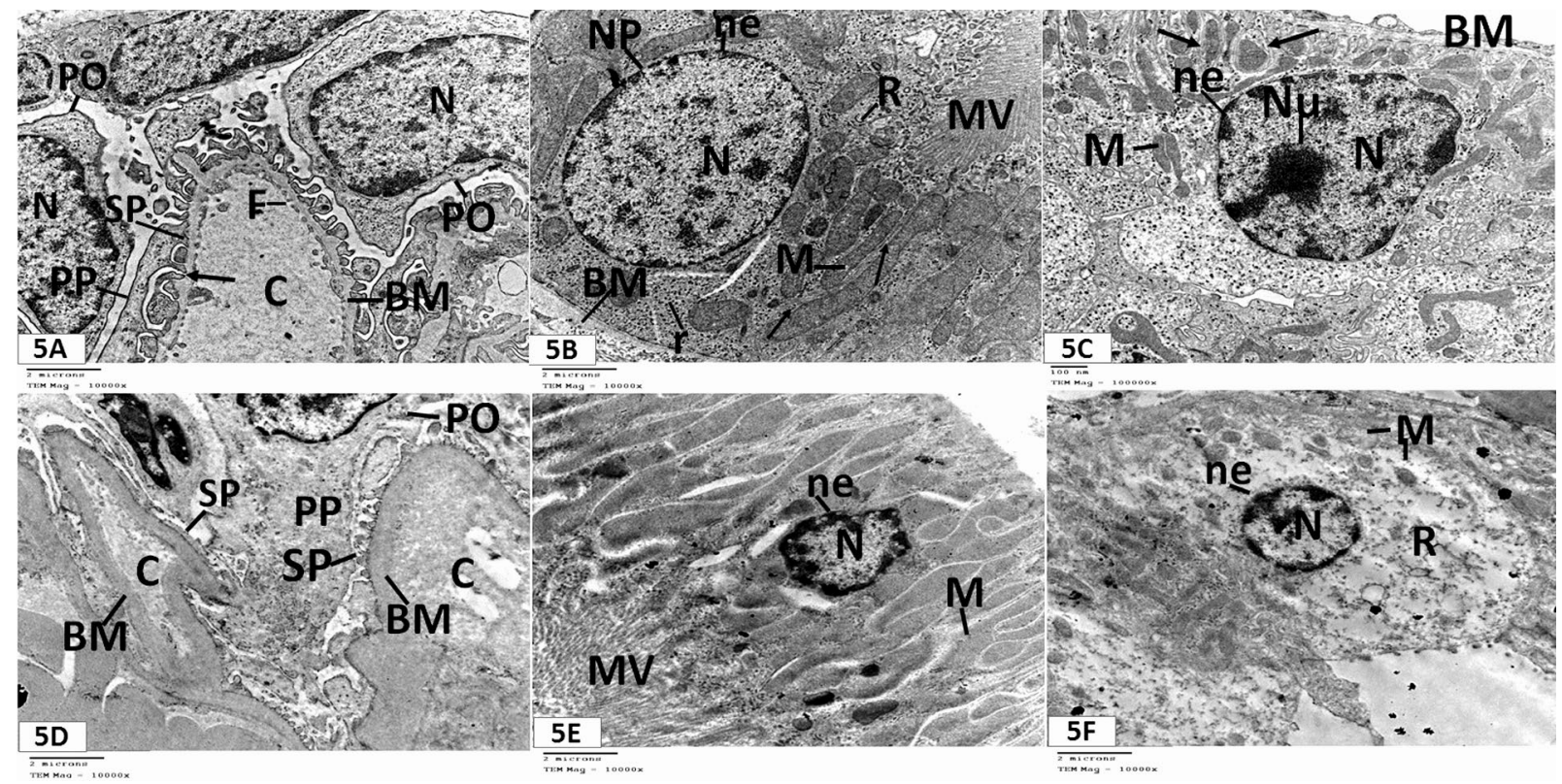

Figure 5. Transmission electron microscopy of the ultrathin renal cortex section (TEM $\times 10,000$ magnification); A. Group I shows a part of renal corpuscle. The glomerular capillary $(C)$, fenestrated endothelium $(F)$, the trilaminar glomerular basement membrane (BM), podocytes (PO) with their nuclei (N), primary cytoplasmic process (PP) and secondary processes (SP); B. Group I shows a part of cell lining the PCT, it has euchromatic nucleus (N), well demarcated nuclear envelope (ne), containing nuclear pores (NP), numerous apical microvilli (MV), basal infoldings (arrow), elongated mitochondria (M), ribosomes $(r)$ and rough endoplasmic reticulum (R); C. Group I showing a part of cell lining the DCT, it has oval nucleus (N), well demarcated nuclear envelope (ne), nucleolus ( $\mathrm{Nu}$ ), basal infoldings (arrow), basement membrane (BM) and longitudinal arranged mitochondria (M); D. Group II shows a part of renal corpuscle, the podocyte (Po), primary processes (PP), distorted and flat secondary foot processes (SP). The basement membrane (BM) lining the glomerular capillaries $(C)$ is thickened and its trilaminar structure is lost; E. Group II showing a part of cell lining the PCT, it has small nucleus (N) with increased heterochromatin, ill demarcated irregular nuclear envelope (ne), less abundant apical microvilli (MV) and elongated mitochondria (M) with loss of their cristae; F. Group II showing a part of cell lining the DCT, it has small nucleus (N) with increased heterochromatin content and irregular ill-demarcated nuclear envelope (ne), loss of basal infoldings, disorganised mitochondria (M) with loss of their cristae, and rarefied cytoplasm (R).

Masson's trichrome stained sections revealed normal distribution of collagen fibres in some elements of renal cortex (Fig. 2C). PAS-stained sections revealed a PAS-positive reaction in some elements of renal cortex (Fig. 3B).

Examination of H\&E stained kidney transverse sections of Group II showed renal corpuscles containing some shrunken glomeruli with wide capsular spaces. Some of the glomerular tuft nuclei were small, irregular and deeply stained. Some of the epithelial cells lining the renal tubules (PCT, DCT and collecting tubules) showed cytoplasmic vacuolisation, intraluminal exfoliation as well as small darkly stained irregular nuclei. The brush border of the some PCT became disrupted (Fig. 1F). Masson's trichrome stained sections showed an apparent increase in the collagen fibres deposition in the parietal layer of Bowman's capsule, around the blood vessels, and in the basal lamina of some renal tubules (Fig. 2F). PAS-stained sections revealed a weak reaction in the parietal layer of Bowman's capsule, in the basal lamina of the renal tubules and in the brush border of the PCT (Fig. 3D).

Electron microscopy. Examination of ultrathin sections of the renal cortex of the Group I (control group) showed that the renal corpuscles was formed of glomerular capillaries lined by fenestrated endothelium and wrapped by podocytes. The podocytes had large irregular euchromatic nuclei, primary processes and secondary processes. Filtration slits were located between the secondary processes. The glomerular basement membrane appeared as a trilaminar structure (Fig. 5A). The epithelial cells lining the PCT were rested on a regular basal lamina that displayed basal infoldings ran perpendicular to it. Numerous rod-like mitochondria were lodged between the basal infoldings and a noticeable number of mitochondria of different sizes and shapes were situated on the upper portion of the cells (Fig. 5B). In addition to mitochondria, the cytoplasm harbours endoplasmic reticulum, ribosomes, Golgi complex 
Table 1. Means and standard deviations (SD) of alanine aminotransferase (ALT), aspartate aminotransferase (AST), urea and creatinine of pregnant rats in Groups' I-II

\begin{tabular}{|c|c|c|c|c|}
\hline \multirow[t]{2}{*}{ Measurements } & \multicolumn{2}{|c|}{ Pregnant rats at 1 day after delivery } & \multicolumn{2}{|c|}{ T test } \\
\hline & $\begin{array}{c}\text { Group I }(n=20) \\
\text { Mean } \pm \text { SD, range }\end{array}$ & $\begin{array}{c}\text { Group II }(n=20) \\
\text { Mean } \pm \text { SD, range }\end{array}$ & $\mathbf{t}$ & $\mathbf{P}$ \\
\hline ALT [IU/L] & $17.2 \pm 2.69 ; 13-22$ & $35.9 \pm 4.817791,31-43$ & 2.144787 & 0.000 \\
\hline AST [IU/L] & $12.6 \pm 1.349897,11-14$ & $26.9 \pm 2.960856,24-31$ & 2.160369 & 0.000 \\
\hline Urea [mg/dL] & $37.39 \pm 1.066094,36-39$ & $49.55 \pm 1.480428,47-51$ & 2.119905 & 0.000 \\
\hline Creatinine [mg/dL] & $0.663 \pm 0.021108,0.64-0.69$ & $0.791 \pm 0.008756,0.78-0.8$ & 2.178813 & 0.000 \\
\hline
\end{tabular}

and lysosomes. The apex of the epithelial cells had tall, closely packed projections called microvilli. The nuclei of these epithelial cells were large, euchromatic with well demarcated nuclear membrane. The nuclei contained clumps of heterochromatin and nucleoli (Fig. 5B). The epithelial cells lining the DCT were resting on a regular basement membrane that exhibited basal infoldings ran perpendicular to it. Numerous elongated mitochondria were lodged between the basal infoldings (Fig. 5C).

Examination of ultrathin sections of the renal cortex of Group II showed histopathological changes. The renal corpuscle revealed thickening of the glomerular basement membrane and loss of its trilaminar structure, some of the secondary foot processes were effaced or fused (Fig. 5D). The epithelial cells lining some PCT had small nuclei with increased heterochromatin contents and irregular ill demarcated nuclear envelope. Their mitochondria were longitudinally arranged with loss of cristae or swollen. Their cytoplasm exhibited primary lysosomes, and their microvilli were less abundant (Fig. 5E).The epithelial cells lining some DCT showed small nuclei with increased heterochromatin. Their cytoplasm exhibited rarefaction, loss of basal infoldings, disorganised mitochondria with loss of their cristae (Fig. 5F).

\section{Biochemical results}

\section{Liver indices}

Statistical analysis of the mean levels of ALT and AST in Groups I and II revealed significant ( $p<0.05$ ) increase in Group II as compared with Group I (Table 1).

\section{Kidney indices}

Statistical analysis of the mean levels of urea and creatinine in Groups I and II revealed significant ( $p<0.05$ ) increase in Group II as compared with Group I (Table 1).

\section{DISCUSSION}

The results of the current study revealed that treatment of pregnant albino rats with levetiracetam induced degenerative changes in their livers, specifically in the form of distortion of the hepatic architecture. Levetiracetam treatment also led to dilatation of the central and the portal veins, cytoplasmic vacuolation and swollen mitochondria with loss of their cristae, such histopathological changes were confirmed by elevation of liver enzymes.

These results are in line with reference no. [31] who reported an episode of acute liver injury immediately following the treatment with levetiracetam in a patient with traumatic brain injury and restoration of normal liver function following stoppage of levetiracetam. Some studies [1,35] reported elevated serum alkaline phosphatase (ALP) level after levetiracetam treatment in children with epilepsy. Some authors observed that the serum ALP level returned to normal after the cessation of levetiracetam administration and concluded that the liver was the main cause of enzyme elevation after exclusion of other causes [35]. Some authors reported an elevation of the liver enzymes in a patient following levetiracetam administration for seizure prophylaxis. The enzymes returned to the normal level after levetiracetam discontinuation [27].

The current study showed that treatment of pregnant albino rats with levetiracetam induced nuclear changes in their liver cells. Such results were confirmed by a study [7] whose authors evaluated the genotoxic effect of levetiracetam (at doses 300 and $600 \mathrm{mg} / \mathrm{kg} \mathrm{bw}$ ) in the liver and brain tissues of treated pregnant rats and their foetuses during pregnancy. They reported a significant increase in DNA damage as detected by alkaline Comet assay, and an appearance of both apoptotic laddered and smeared DNA in the tissues tested. 
On the other hand, the results of the present study were contrary to study whose authors evaluated the levetiracetam tolerability in 14 patients with liver disease and epilepsy. After 6 months of supervision, they reported that none of the patients showed decline of liver function on the base of blood analysis [4]. Based on these observations, they assumed that levetiracetam was an agreeable treatment option in epileptic cases with liver impairment. The results of the present study were also contrary to some authors [17] who reported that chronic administration of levetiracetam to rats at a therapeutic dose ( $54 \mathrm{mg} /$ day) revealed no histopathological differences in their liver as compared to those of control. Some authors [34] mentioned that levetiracetam did not interact with the cytochrome P450 system and was the best drug of choice for cases with liver impairment.

In the current study, Masson's trichrome staining showed higher level of collagen-fibre distribution around the central vein and the elements of the portal tract in levetiracetam treated group as compared with sections from the control group. Some authors mentioned that excessive collagen deposition in the liver was mediated by death of hepatocytes and activation of hepatic stellate cells. They added that lipid peroxidation including the generation of reactive oxygen species, could be implicated as a cause of excessive collagen deposition [29].

The current study revealed that treatment of pregnant albino rats with levetiracetam induced degenerative changes in their renal cortex. The changes were in the form of widening of the Bowman's spaces, pyknosis of some of the glomerular tuft nuclei, thickening and disruption of the glomerular basement membrane, and fusion and effacement of secondary foot processes. Levetiracetam treatment also led to cytoplasmic vacuolisation, intraluminal exfoliation, and disorganised mitochondria in the cells lining the renal tubules. In addition, levetiracetam treatment led to an increased heterochromatin contents of some nuclei of the cells lining the renal tubules, such pathological changes were confirmed by significant elevation of the levels of urea and creatinine.

These findings were in line with a study [14] whose authors presented occurrence of acute kidney injury in Drug Reaction with Eosinophilia and Systemic Symptoms (DRESS) 2 months after starting levetiracetam to a 75-year-old man with glioblastoma. Also, the results of the present study were in line with some researchers [30] who reported occurrence of an acute kidney injury 1 day after the initiation of levetiracetam treatment for new-onset seizures in a healthy 23-year-old female patient. Based on the time course of the patient's elevation in the level of serum creatinine and the exclusion of other causes, the authors suggested that levetiracetam treatment was contributed to the acute kidney injury.

On the other hand, the results of the current study were contrary to some researchers [13] who evaluated the clinical use of intravenous levetiracetam in patients with renal impairment and didn't detect any major side effects any patients. So, they concluded that intravenous levetiracetam treatment was effective and safe in patients with renal impairment. The results of the present study also were contrary to a study [19] whose authors presented two paediatric cases of levetiracetam overdose for 1 month (115 and $300 \mathrm{mg} / \mathrm{kg} / \mathrm{day}$ ) and didn't observe remarkable severe side effects in their patients. So, they suggested that accidental treatment with an overdose of levetiracetam in children for long period was rarely accompanied with serious side effects.

In the current work, Masson's trichrome staining showed higher level of collagen-fibre distribution in the parietal layer of Bowman's capsule, and in the wall of some renal tubules in levetiracetam treated group as compared with sections from the control group. Such results may be caused by disturbance in oxidant/ antioxidant system. Such suggestion was supported by some authors who investigated the association of reactive oxygen species/antioxidant systems with kidney fibrosis following ischaemic/reperfusion injury [12]. They concluded that reactive oxygen species/ /oxidative stress was contributed to the progression of kidney fibrosis [12].

In the current study, the degenerative changes that were observed in the liver and kidney might be due to disturbance in the oxidant/antioxidant ratio. Such suggestion was supported by researchers [7] who explained that genotoxic potential of levetiracetam by decreased free radical neutralisation and/ or an increased production of free radicals.

\section{CONCLUSIONS}

Levetiracetam induced deleterious effects on the liver and kidney of pregnant albino rats. Continuous assessment of the liver and kidney functions during levetiracetam therapy is advised. In addition, further investigations are recommended to clarify the mechanism of levetiracetam toxicity. 


\section{REFERENCES}

1. Attilakos A, Dinopoulos A, Paschalidou M, et al. Effect of Levetiracetam Monotherapy on Liver Enzymes and Creatine Kinase Concentrations in Children with Epilepsy: A Prospective Study. J Clin Neurol. 2018; 14(4): 594-595, doi: 10.3988/ jcn.2018.14.4.594, indexed in Pubmed: 30284772.

2. Bancroft JD, Gamble M. Theory and practice of histological techniques. 6th ed. Pbl. Churchill Livingston, Edinburg 2008: 340-348.

3. Bansal R, Suri V, Kumar P, et al. Levetiracetam use during pregnancy in women with epilepsy: Preliminary observations from a tertiary care center in Northern India. Indian J Pharmacol. 2018; 50(1): 39-43, doi: 10.4103/ijp. IJP 692 17, indexed in Pubmed: 29861526.

4. Bilo L, Meo R, de Leva MF, et al. Levetiracetam in patients with epilepsy and chronic liver disease: observations in a case series. Clin Neuropharmacol. 2008; 31(4): 221-225, doi: 10.1097/WNF.0b013e31815c1d92, indexed in Pubmed: 18670245

5. Broli M, Provini F, Naldi I, et al. Unexpected gamma glutamyltransferase rise increase during levetiracetam monotherapy. Epileptic Disord. 2010; 12(1): 81-82, doi: 10.1684/ epd.2010.0291, indexed in Pubmed: 20159672.

6. Ding SP, Li JC, Jin C. A mouse model of severe acute pancreatitis induced with caerulein and lipopolysaccharide. World J Gastroenterol. 2003; 9(3): 584-589, doi: 10.3748/ wjg.v9.i3.584, indexed in Pubmed: 12632523.

7. EL-Shorbagy HM, Hamdi H. Genotoxic and mutagenic studies of the antiepileptic drug levetiracetame in pregnant rats and their fetuses. Int J Pharm Sci. 2016; 8(2): 82-88.

8. Fossati P, Prencipe L, Berti G. Enzymic creatinine assay: a new colorimetric method based on hydrogen peroxide measurement. Clin Chem. 1983; 29(8): 1494-1496, indexed in Pubmed: 6872208.

9. Harden CL, Meador KJ, Pennell PB, et al. Practice parameter update: management issues for women with epilepsy--focus on pregnancy (an evidence-based review): teratogenesis and perinatal outcomes: report of the Quality Standards Subcommittee and Therapeutics and Technology Assessment Subcommittee of the American Academy of Neurology and American Epilepsy Society. Neurology. 2009; 73(2): 133-141, doi: 10.1212/WNL.0b013e3181a6b312, indexed in Pubmed: 19398681.

10. Hayat MA. Principles and techniques of electron microscopy: biological applications. 4th ed. Cambridge University Press, USA 2000: 4-85.

11. Institute for Laboratory Animal Research. Committee for the Update of the Guide for the Care and Use of Laboratory Animals, National Research Council, Guide for the Care and Use of Laboratory Animals. 8th ed., National Academy Press, Washington, DC. 2011: 11-132.

12. Kim J, Seok YMi, Jung KJ, et al. Reactive oxygen species/oxidative stress contributes to progression of kidney fibrosis following transient ischemic injury in mice. Am J Physiol Renal Physiol. 2009; 297(2): F461-F470, doi: 10.1152/ ajprenal.90735.2008, indexed in Pubmed: 19458120.

13. Lapmag A, Lertsinudom S, Chaiyakam A, et al. Clinical outcomes of intravenous levetiracetam treatment in patients with renal impairment. Neurol Int. 2018; 10(3): 7469 , doi: 10.4081/ni.2018.7469, indexed in Pubmed: 30344963.

14. Leblanc M, Plaisance M. Levetiracetam-associated acute kidney injury and drug reaction with eosinophilia and systemic symptoms (DRESS) syndrome. J Nephrol. 2014; 4(4): 152-155, doi: 10.4236/ojneph.2014.44022.

15. Lyseng-Williamson KA. Levetiracetam: a review of its use in epilepsy. Drugs. 2011; 71(4): 489-514, doi: 10.2165/11204490-000000000-00000, indexed in Pubmed: 21395360.

16. Neves EG, Ramos-Perez FM, Freitas DQ, et al. Radioprotective effect of sodium selenite on developing teeth. Braz Dent J. 2013; 24(4): 375-379, doi: 10.1590/01036440201302193, indexed in Pubmed: 24173260.

17. Omer H, Kutb M. Chronic histopathological effects of levetiracetam on some internal organs of adult albino rats.
Egypt J Forensic Sci. 2015; 5(2): 41-45, doi: 10.1016/j. ejfs.2014.06.002.

18. Orsonneau JL, Massoubre C, Cabanes M, et al. Simple and sensitive determination of urea in serum and urine. Clin Chem. 1992; 38(5): 619-623, indexed in Pubmed: 1582010.

19. Öztürk Z, Havalı C, Serdaroğlu A, et al. Two cases of levetiracetam overdose without any serious side effects. Gazi Med J. 2016; 28(2): 137-138.

20. Paget GC, Barens JM. Toxicity in evaluation of drug activities. pharmaceutic. Lournace and Bacharachai Academic Press, London, New York 1964: 1-13.

21. Pipkin BF. Medical Statistics made easy. 5th ed. Churchill Livingstone, New York 1984: 46-56.

22. Patel SI, Pennell PB. Management of epilepsy during pregnancy: an update. Ther Adv Neurol Disord. 2016; 9(2): 118-129, doi: $10.1177 / 1756285615623934$, indexed in Pubmed: 27006699.

23. Porter J, Rogawski MA. Antiseizure Drugs. In: Katzung BG, Masters SB, Trevor AJ. Basic and Clincal Pharmacology. 12th ed. McGraw-Hill Companies 2012: 409-424.

24. Rang HP, Ritter JM, Flower RJ. Rang and Dale's Pharmacology. Eighth ed. Pbl Elsevier Ltd. 2016: 559-569.

25. Rey JA. Antipsychotic Drugs. In: Whalen K, Finkel R, Panavelil TA. Lippincott Illustrated Reviews: Pharmacology. 6th ed. Wolters Kluwer 2015: 147-157.

26. Rogawski MA, Bazil CW. New molecular targets for antiepileptic drugs: alpha(2)delta, SV2A, and K(v)7/KCNQ/M potassium channels. Curr Neurol Neurosci Rep. 2008; 8(4): 345-352, indexed in Pubmed: 18590620.

27. Sethi NK, Sethi PK, Torgovnick J, et al. Asymptomatic elevation of liver enzymes due to levetiracetam: a case report. Drug Metabol Drug Interact. 2013; 28(2): 123-124, doi: 10.1515/dmdi-2013-0006, indexed in Pubmed: 23420283

28. Sharma U, Pal D, Prasad R. Alkaline phosphatase: an overview. Indian J Clin Biochem. 2014; 29(3): 269-278, doi: 10.1007/s12291-013-0408-y, indexed in Pubmed: 24966474

29. Shimizu I, Shimamoto N, Saiki K. et al., Lipid Peroxidation in Hepatic Fibrosis. In: Catala, A. Lipid Peroxidation. 1st ed. Intechopen 2012: 483-492.

30. Spengler DC, Montouris GD, Hohler AD. Levetiracetam as a possible contributor to acute kidney injury. Clin Ther. 2014; 36(8): 1303-1306, doi: 10.1016/j.clinthera.2014.06.002, indexed in Pubmed: 24986483.

31. Syed A, Adams C. Acute liver failure following levetiracetam therapy for seizure prophylaxis in traumatic brain injury. Case Reports Clin Med. 2012; 1(2): 42-45, doi: $10.4236 / \mathrm{crcm} .2012 .12010$.

32. Thomas L. Alanine aminotransf-erase (ALT), aspartate aminotrasferase (AST), in Thomas, L editor. Clinical Lab-oratory Diagnostics. 1st ed. TH-Books Verlagsgesellschaft, Frankfurt 1998: 55-56.

33. Tomson T, Battino D. Teratogenic effects of antiepileptic medications. Neurol Clin. 2009; 27(4): 9931002, doi: 10.1016/j.ncl.2009.06.006, indexed in Pubmed: 19853219

34. Vidaurre J, Gedela S, Yarosz S. Antiepileptic drugs and liver disease. Pediatr Neurol. 2017; 77: 23-36, doi: 10.1016/j.pediatrneurol.2017.09.013, indexed in Pubmed: 29097018.

35. Xiong N, Hou L, Lu Na, et al. Probable levetiracetam-related serum alkaline phosphatase elevation. BMC Neurol. 2012; 12: 97, doi: 10.1186/1471-2377-12-97, indexed in Pubmed: 22994584.

36. Yang H, Lavu S, Sinclair DA. Nampt/PBEF/Visfatin: a regulator of mammalian health and longevity? Exp Gerontol. 2006; 41(8): 718-726, doi: 10.1016/j.exger.2006.06.003, indexed in Pubmed: 16842957.

37. Zhu F, Lang SY, Wang XQ, et al. Long-term effectiveness of antiepileptic drug monotherapy in partial epileptic patients: a 7-year study in an epilepsy center in china. Chin Med J. 2015; 128(22): 3015-3022, doi: 10.4103/03666999.168968, indexed in Pubmed: 26608980. 